\title{
Arabidopsis thaliana microRNA162 level is posttranscriptionally regulated via splicing and polyadenylation site selection
}

\author{
Maria Barciszewska-Pacak, Katarzyna Knop, Artur Jarmołowski and \\ Zofia Szweykowska-Kulińska
}

Department of Gene Expression, Institute of Molecular Biology and Biotechnology, Faculty of Biology, Adam Mickiewicz University in Poznań, Poznań, Poland

\begin{abstract}
Arabidopsis microRNA162 (miRNA162) level regulation was studied under abiotic stresses, such as drought and salinity. The $\operatorname{TaqMan}^{\circledR}$ microRNA assay proved that A. thaliana miRNA162 level was elevated under these stresses, confirming its salt and drought responsiveness. The promoter region analyses of $A$. thaliana miRNA162a and $b$ genes (MIR162a and MIR162b) identified numerous salinity and drought responsive elements. However, our results indicated that Arabidopsis MIR162a was presumably the main locus responsible for the mature ath-miRNA162 accumulation under the stresses tested, and the MIR162b was generally rather weakly expressed, both in control and under the stress conditions. The MIR162a structure was confirmed to be complex and the pri-miRNA162a hairpin structure was shown to span an alternative exon and an intron. The MIR162a transcription generated a few pri-miRNA162a splicing isoforms that could be functional and non-functional. Upon drought and salinity stresses, the regulation of the pri-miRNA162a alternative splicing pattern revealed an increase of a functional pri-miR162a isoform and a preferential distal polyA site selection under the stress conditions. Apart from the potential transcriptional regulation of the miRNA genes (MIRs) expression, the data obtained point to an essential role of posttranscriptional regulation of Arabidopsis microRNA162 level.
\end{abstract}

Key words: miRNA, pri-miRNA, abiotic stress, gene expression

Received: 03 June, 2016; revised: 05 July, 2016; accepted: 11 July, 2016; available on-line: 05 October, 2016

\section{INTRODUCTION}

Plant miRNAs are important general negative regulators of gene expression. They are short, single stranded, noncoding RNAs that act at the posttranscriptional level and guide target mRNA cleavage or translation inhibition (Bartel 2004; Brodersen et al., 2008; Beauclair et al., 2010). As the products of action of RNA Pol II, the miRNA gene (MIR) primary transcripts (pri-miRNAs) are 5' capped and 3' polyadenylated (Xie et al., 2005). They contain a characteristic stem and loop structure in which microRNA and its cognate, the so called microRNA star $\left(^{*}\right)$, are embedded. The plant pri-miRNAs' processing occurs in the nucleus where an RNAse III-type enzyme, DICER LIKE 1 (DCL1), is responsible for all their endonucleolytic cleavages leading to the formation of pre-miRNAs, further cleaved by the same enzyme to produce the microRNA and microRNA* duplexes (Park et al., 2002). However, an efficient biogenesis of plant miRNAs requires the involvement of plenty of proteins (Vazquez et al., 2004; Kurihara et al., 2006; Dong et al., 2008; Laubinger et al., 2008; Manavella et al., 2012; Ren et al., 2012; Zhan et al., 2012; Kruszka et al., 2013; Rogers \& Chen, 2013; Wang et al., 2013), from which DCL1, HYPONASTIC LEAVES 1 (HYL1) (Han et al., 2004; Vazquez et al., 2004), and SERRATE (SE) (Lobbes et al., 2006; Yang et al., 2006; Dong et al., 2008) appear to be the most crucial. The complex plant miRNAs' biogenesis is mainly due to the structure of MIR genes. The promoter regions of many plant microRNA genes contain numerous abiotic response elements (RE) found using bioinformatic tools (Higo et al., 1999; Megraw et al., 2006; Zhao et al., 2013). Half of the plant miRNA gene bodies of independent transcription units possess introns (Szarzynska et al. 2009; Rogers and Chen, 2013; Szweykowska-Kulinska et al., 2013; Kruszka et al., 2013; Kruszka et al., 2014; Zielezinski et al., 2015) and the Arabidopsis pri-miRNAs were found to contain many alternative polyadenylation signals (Szarzynska et al., 2009; Bielewicz et al., 2012; Bielewicz et al., 2013; Schwab et al., 2013; Zielezinski et al., 2015). Additionally, 29 Arabidopsis miRNAs characterized so far are embedded within the introns of other genes encoding proteins or noncoding RNAs (Brown et al., 2008; Yan et al., 2012; Zielezinski et al., 2015). Therefore, not only transcriptional regulation of at least some microRNA gene expression must exist but also the constitutive and alternative splicing and polyadenylation processes can indeed posttranscriptionally regulate the miRNA level (Bielewicz et al., 2012; Bielewicz et al., 2013; Yan et al., 2012; Jia et al., 2013).

Among the first identified plant miRNAs from Arabidopsis (Reinhart et al., 2002; Jones-Rhodes et al., 2004), miRNA162 was found to be encoded by two genes: MIR162a and MIR162b, respectively. MIR162b is intronless (At5g23065) while the MIR162a gene contains multiple introns and its transcript undergoes a complex constitutive and alternative splicing (Reinhart et al., 2002; Hirsch et al., 2006; Brown et al., 2008). The pre-miRNA162a is located in the intron 2 of the protein coding At5g08185 gene. Hirsch et al. in 2006 reported the identification of four MIR162a splice isoforms of which only one is functional and can give rise to miR162 production (Hirsch et al., 2006), while Brown and coworkers in 2008 characterised five splice iso-

e-mail: zofszwey@amu.edu.p

Accession numbers: The data used in this study have been deposited under NCBI accession number KX228747.

Abbreviations: ath-miRNA, Arabidopsis thaliana miRNA; MIR, miRNA gene 
forms of which two are potentially functional and can give rise to mature miRNA (Brown et al., 2008). Both ath-miRNA162a and ath-miRNA162b have identical nucleotide sequences and it is known that ath-miR162 targets the DCL1 mRNA (Xie et al., 2003). Arabidopsis miR162 can be detected in a mir162a mutant which suggests that the MIR162b gene can compensate for the loss-of-function of MIR162a (Hirsch et al., 2006). Arabidopsis MIR162b transcript appeared to have at least two alternative 3' ends, while MIR162a was not reported to have alternative polyA sites (Hirsch et al., 2006).

A relationship between splicing and processing of an intronic pri-miRNA in Arabidopsis plants has been studied in the case of miR162a and its host gene (Hirsch et al., 2006; Brown et al., 2008). Here, we show the posttranscriptional regulation of miR162 abundance in Arabidopsis thaliana in control and two abiotic stress conditions: 20\% soil water content (SWC) drought and salinity. The data presented point to the role of splicing and polyadenylation already at the pri-miRNA level during the intronic miRNA162a biogenesis. They confirm the posttranscriptional regulation of ath-miRNA162 under both abiotic stresses applied. This regulation affects the miRNA stress responsiveness and therefore the ability of plants to resist the stress.

\section{MATERIALS AND METHODS}

Plant material and growth conditions. Arabidopsis thaliana (L.) Heynh, Col-0 wild type plants were grown and stressed as previously described (Barciszewska-Pacak et al., 2015). The control and both stress experiments were done in three biological replicates. The stress application was confirmed by the amplification of stress marker mRNAs (Barciszewska-Pacak et al., 2015).

RNA isolation. For semi-quantitative RT-PCR and quantitative real-time PCR analyses, including TaqMan ${ }^{\circledR}$ miRNA assays (ABI, Life Technologies, USA), total RNA was isolated from $100 \mathrm{mg}$ of 15-day old seedlings and 1.13 growth stage (Boyes et al., 2001) plant leaves, and assessed with regard to the RNA concentration, RNA integrity determination, and DNA contamination removal, as previously described (Szarzynska et al., 2009; Barciszewska-Pacak et al., 2015).

Quantitative real-time PCR profiling of miRNAs and the pri-miRNA splicing and polyA isoforms. Three $\mu \mathrm{g}$ and $10 \mathrm{ng}$ of DNA-free RNA were used for real-time PCR and TaqMan ${ }^{\circledR}$ miRNA assay (ABI, Life Technologies, USA) analyses, respectively, carried out as described in (Kruszka et al., 2013; Barciszewska-Pacak et al., 2015). All measurements were performed in three biological replicates. The list of qPCR primers for splicing and polyA transcript isoforms, as well as the TaqMan ${ }^{\circledR}$ miRNA assay probes can be found in the supplementary data Table S1 (at www.actabp.pl). The amplification efficiency of each primer pair (200 nM each, final concentration) was calculated by making a 2 -fold dilution series of the mix of templates, calculating a linear regression based on the data, and estimating the efficiency from the line slope. Only primer pairs with the highest and almost equal efficiency (max. difference of $1 \%$ was approved) were used for analyses.

pri-miRNA 5' and 3' RLM-RACE experiments. The 5' and 3' RLM-RACE experiments were performed using a GeneRacer ${ }^{\mathrm{TM}}$ Kit (Invitrogen ${ }^{\mathrm{TM}}$ Life Technologies, Carlsbad, CA, USA), according to the manufacturer's protocol. The 5' and 3' RLM-RACE PCRs were done using an Advantage ${ }^{\circledR} 2$ PCR Enzyme Sys- tem (Clontech, Mountain View, CA, USA), according to the manufacturer's protocol. All primers used in the experiments are listed in Table S1 (Supplementary data at www.actabp.pl). Cloning and sequencing of 5' and 3' RLM-RACE products were done as previously reported in Kruszka et al., 2013.

\section{RESULTS}

\section{Drought and salt stress upregulation of Arabidopsis thaliana miRNA162}

We have previously shown by using a high throughput real-time PCR platform, mirEX (modified mirEX platform, Bielewicz et al., 2012, http://comgen.pl/ mirex2/; Zielezinski et al., 2015) that Arabidopsis thaliana pri-miRNA162a and $\mathrm{b}$ were both down-regulated under $20 \%$ SWC drought stress and their expression was not changed under a salinity stress (Barciszewska-Pacak et al., 2015). Small RNA next generation sequencing (NGS) results had shown a slight elevation of ath-miRNA162 level that was almost statistically significant $(\not$-values $=0.055$ and 0.06) under the drought and salinity stress conditions (Barciszewska-Pacak et al., 2015). We thus decided to verify the NGS data by applying a sensitive TaqMan ${ }^{\circledR}$ miRNA assay (ABI, Life Technologies, USA), and show that indeed the level of $A$. thaliana miRNA162 is 2.1and 2.9-fold increased under drought and salinity stresses, respectively $(p$-value $<0.01)$ (Fig. 1). These results confirm the Arabidopsis thaliana miRNA162 responsiveness to drought and salt stresses, which has not been shown so far.

\section{Transcriptional regulation of Arabidopsis thaliana MIRNA162 $a$ and $b$ expression}

Drought and salinity responsiveness of Arabidopsis miR162 prompted us to investigate the potential transcriptional regulation of the miRNA genes under the stress conditions. For this purpose, we examined the promoter regions of both, $A$. thaliana MIR162a and MIR162b. The Database of Plant Cis-acting Regulatory DNA Elements (Higo et al., 1999) has revealed the presence of six MYB motifs (MYBST1, MYBCORE, two MYB1AT, MYBGAHV, MYBPLANT) and five MYC motifs (MYCCONSENSUSAT type) in the two
A

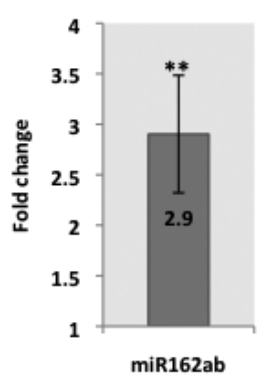

B

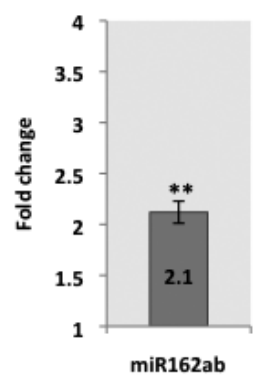

Figure 1. Quantification of $A$. thaliana miRNA162a, b levels under salinity and drought stresses.

(A) MiRNA162a, b level detection under salinity stress revealed by TaqMan ${ }^{\circledR}$ miRNA assay. (B) MiRNA162a, b level detection under $20 \%$ SWC drought stress revealed by TaqMan ${ }^{\circledR}$ miRNA assay. Grey bars depict the fold change of miRNA levels under the stresses when compared to the control conditions. Values on the charts are shown as the mean \pm S.D. fold change from three independent experiments. Double stars depict a two-tailed Student's $t$-test $(p \leq 0.01)$ suggested statistically significant results. 
kilobase (kb) MIR162a potential promoter region. In the case of $2 \mathrm{~kb} M I R 162 b$ promoter region, the same database indicated seven MYB motifs (MYBCORE, two MYBGAHV, two MYBPLANT, MYB26PS, MYBCOREATCYCB1), and three MYC (MYCCONSENSUS type) motifs. Additionally, the MIR162a promoter region contains four ABRE (ACGT-containing abscisic acid response element)-like sequences (three ACGTATERD1 type, one ABRELATERD1 type) required for etiolation-induced expression of Arabidopsis erd1 (early responsive to debydration 1$)$. The MIR162b promoter region also possesses one ABRE (ACGTATERD1) and one DRE (DRE2COREZMRAB17) motif. All of the above motifs refer to regulation of expression of genes that are responsive to the water stress in Arabidopsis (Higo et al., 1999), suggesting a potential of transcriptional regulation of A. thaliana MIR162a and $b$ expression under drought stress. Based on the fact that salinity, drought, and also cold, elicit many common and interactive downstream effects (Liu et al., 2007), we examined the MIR162a and $b$ promoter regions with regard to salinity responsiveness searching for different responsive elements that were reported to play important roles in the plant responses to salinity, drought, osmotic stress, and reactive oxygen species (ROS) stress. We have found the already mentioned DRE element in the MIR162b promoter that is a cis-acting promoter element known to regulate gene expression in response not only to drought but also salt and cold stresses in Arabidopsis (Cheng et al., 2013). The MIR162a promoter also contains a single GCCCORE motif that is the ethylene-responsive element, and Ethylene Response Factor1 (ERF1) is often considered as the one related to the salt stress response through ethylene signalling in Arabidopsis (Cheng et al., 2013). Within the same promoter, a GT1GNSCAM4 motif was found, as GT1 is known to be the RE present in pathogen and salt-induced genes of Glycine max (Higo et al., 1999). As drought and salt stresses activate the dehydration response element binding factor 2 (DREB2) and elevate abscisic acid (ABA) levels (Liu et al., 2007), the DRE response element, and the above ABRE and MYC/MYB responsive elements can be potentially recognized in plants under the salt and drought stresses, suggesting a potential transcriptional regulation of both ath-miR162 genes under stress conditions studied.

\section{Posttranscriptional regulation of Arabidopsis thaliana MIRNA162 $a$ and $b$ expression}

The discrepancy between the levels of Arabidopsis pri-miRNAs 162a and b (Barciszewska-Pacak et al., 2015), and the mature miRNAs $162 \mathrm{a}$ and $\mathrm{b}$ in the course of the plant's response to both, drought and salt stresses, encouraged us to investigate the possible posttranscriptional ways of regulation of the miRNAs abundance under the stress conditions. Firstly, we confirmed an already described for wild type (wt) Arabidopsis plants MIR162a splicing pattern (Hirsch et al., 2006) in 15 dayold seedlings under control and salt stress conditions, as well as in 30 day-old adult plants under control and $20 \%$ SWC drought conditions (Figs. 2A, 3). With regard to the miRNA162a production, the differentially spliced MIR162 a transcripts were called: a, b, c, d (Hirsch et al., 2006), as functional (a) and non-functional: b, c, d, respectively. The functional splicing isoform is the splicing variant retaining all introns, where the pre-miRNA162a hairpin's left arm is formed by an alternative exon 3 containing miRNA162a-5p, and the right arm - by a retained intron 3 containing miRNA162a-3p. All nonfunctional splicing variants of pri-miRNA162a represent transcripts lacking either exon 3 or intron 3 , or both of them (Fig. 3). For the A. thaliana MIR162b gene, we also confirmed the lack of any introns in the gene body (Figs. 2B, 3), as it was shown previously (Hirsch et al., 2006). However, 5'RLM-RACE experiments for
A

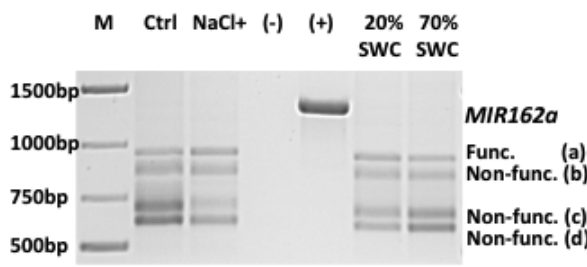

B

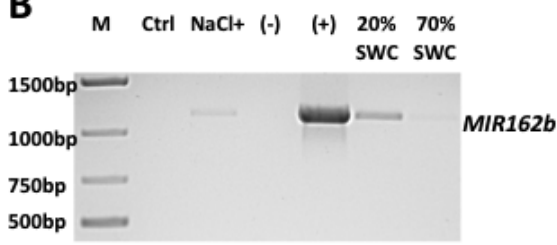

C

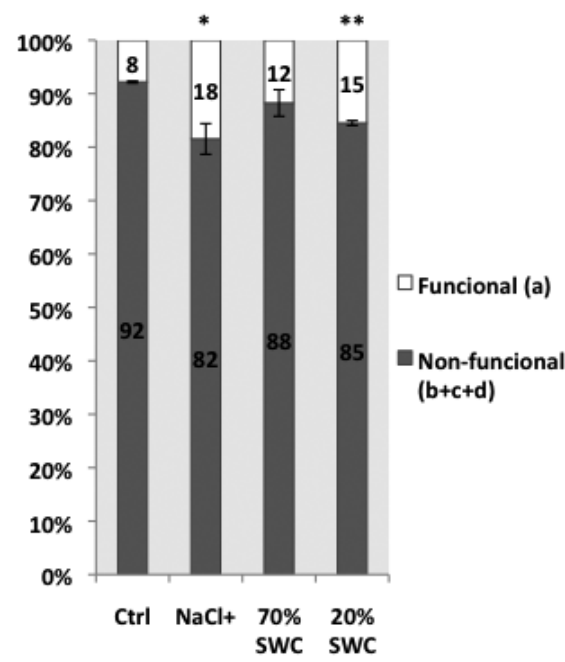

Figure 2. A. thaliana MIR162a and MIR162b transcript analyses and quantification of pri-miRNA162a splicing isoforms under salinity and drought stresses.

(A) RT-PCR detection of: Functional (a) and Non-functional (b, c, d) MIR162a transcripts in control (Ctrl) and salinity ( $\mathrm{NaCl}+$ ) stressed seedlings, and 70\% SWC (control) and 20\% SWC (drought) treated adult plants. (-) and (+) depict negative and positive (genomic DNA) controls, respectively. M depicts $1 \mathrm{~Kb}$ Plus DNA Ladder (Thermo Fisher Scientific). (B) RT-PCR detection of MIR162b transcript in control (Ctrl) and salinity ( $\mathrm{NaCl}+$ ) stressed seedlings, and $70 \%$ SWC (control) and 20\% SWC (drought) treated adult plants. (-) and (+) depict negative and positive (genomic DNA) controls, respectively. M depicts 1 Kb Plus DNA Ladder (Thermo Fisher Scientific). (C) Pri-miRNA162a splicing functional (a) (white bars) and non-functional $(\mathbf{b}+\mathbf{c}+\mathbf{d})$ (grey bars) isoform level detections under salinity and drought stresses revealed by RT-qPCR. The bars depict the percentage of particular splicing isoforms. Values on the charts are shown as the mean \pm S.D. percentage from three independent experiments. Single and double stars depict a two-tailed Student's $t$-test $(p \leq 0.05)$ and $(p \leq 0.01)$ suggested statistically significant results, respectively. 


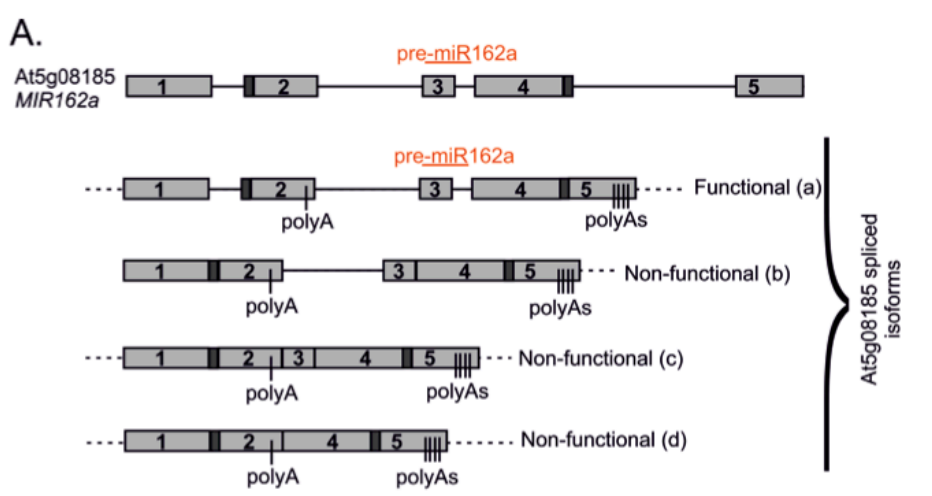

B.

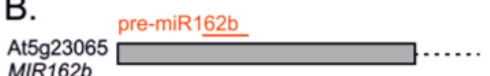

MIR162b

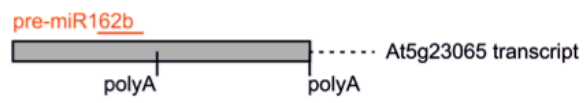

Figure 3. Schematic representation of MIR162a (At5g08185), MIR162b (At5g23065) genes, and the differentially spliced: functional (a) and non-functional (b, c, d) transcripts of MIR162a.

Grey boxes represent the numbered exons, black horizontal lines between exons represent introns, dark grey boxes depict $5^{\prime}$ and $3^{\prime}$ untranslated regions (UTRs). The polyadenylation site selection events are depicted by short, vertical lines named polyA or polyAs and pre-miR162a location is visualised by red, short horizontal lines above a particular gene and mRNA region.

control and salt-stressed seedlings enabled us to determine 134 nucleotides (nt) longer 5'end of the MIR162b transcript (KX228747) when compared to the 5'end described earlier by Hirsch and coworkers (2006). Under the same conditions, 3'RACE experiments performed on the 5'RLM-RACE derived cDNA led us to define a 298 nt longer 3'end of the MIR162b transcript (KX228747), when compared to the 3'end defined by the already mentioned research of Hirsch and coworkers (2006).
A

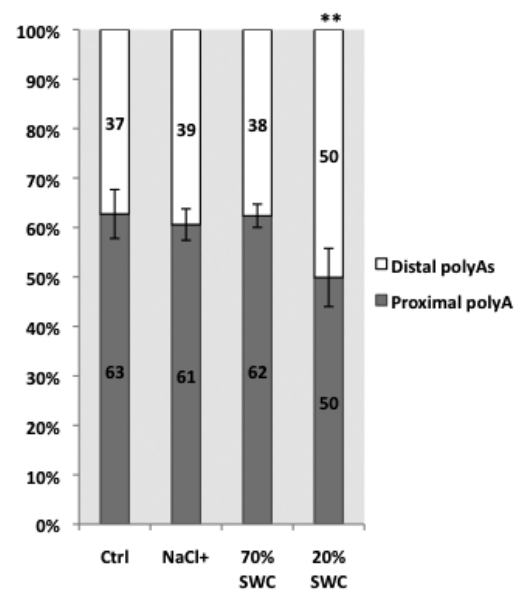

B

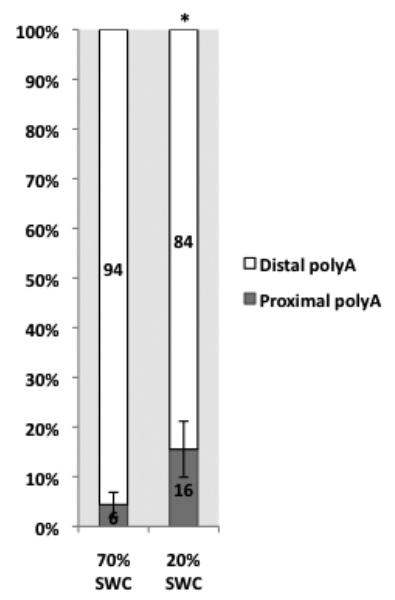

Figure 4. Quantification of $A$. thaliana pri-miRNA162a and pri-miRNA162b polyadenylation isoforms under salinity and drought stresses.

(A) Pri-miRNA162a polyadenylation isoform level detections under salinity and drought stresses revealed by RT-qPCR. (B) Pri-miRNA162b polyadenylation isoform level detections under drought stress revealed by RT-qPCR. The white and grey bars depict the percentage of distal and proximal polyA isoforms, respectively. Values on the charts are shown as the mean \pm S.D. percentage from three independent experiments. Single and double stars depict a two-tailed Student's $t$-test $(p \leq 0.05)$ and $(p \leq 0.01)$ suggested statistically significant results, respectively.
We then quantitatively evaluated the level of the MIR162a functional and non-functional splicing isoforms in control, salt, and drought stress treated Arabidopsis plants by RT-qPCR (Fig. 2C). The functional primiRNA162a splicing isoform level increased more than two fold under the salt stress and slightly (1.25 fold) under the $20 \%$ SWC drought stress. These changes were accompanied by detection of decreased levels of nonfunctional pri-miRNA162a splicing isoforms (Fig. 2C). These results pinpointed a significant splicing pattern impact of MIR162a transcript on mature ath-miRNA162a production.

The 3'RACE MIR162a and MIR162b experiments performed on the control and stressed seedlings revealed numerous polyadenylation site selection events in these MIR transcripts. We have grouped and named them as proximal (located in the MIR162a exon 2 and the middle part of MIR162b) and distal (located in the last MIR162a exon 5 and at the end of MIR162b) polyadenylations (polyAs) (Fig. 3). We quantified the MIR162a and MIR162b polyA isoforms under control and both stress conditions by RT-qPCR (Fig. 4). Distal MIR162a polyAs selection increased under drought when compared to the control, while the polyA site selection was not affected under the salinity stress (Fig. 4). It is important to realize that only distal polyA site selection leads to the production of a functional pri-miR162a splice isoform (see Fig. 3). In the case of the MIR162b transcripts, proximal polyA site selection increased under drought when compared to the control conditions (Fig. 4), while under control and salinity conditions the distal polyA isoform was predominant, and the proximal polyA site selection was barely detectable (data not shown). The polyadenylation analyses of MIRs: $162 a$ and $b$ transcripts revealed how important it is to consider the process of polyadenylation with regard to the plant miRNA gene expression regulation under both, the control and stress conditions.

\section{DISCUSSION}

MicroRNA162 is known to be stress responsive in many plant species, although it is not reported as such in Arabidopsis. Its level was reported to be elevated in salt stressed maize roots (Ding et al., 2009) and it also belongs to hypoxia responsive maize miRNA families (Zhang et al., 2008). It is responsive to mechanical stress in poplar ( $\mathrm{Lu}$ et al., 2005). It is drought and salt responsive in cellulosic biofuel crop switchgrass (Sun et al., 2012). It also responds to drought in rice (Zhou et al., 2010) and to non-essential metals, like cadmium in rice and rapeseed, aluminum in Nicotiana tabacum and mercury in Medicago truncatula (Gielen et al., 2012). Here we present data showing Arabidopsis thaliana mature miRNA162 responding to drought and salinity stresses that severely threaten plant growth and crop productivity worldwide. As miRNA162 regulates DCL1 
expression in plants, its particular stress responsiveness may potentially have a significant impact on the plant miRNA biogenesis within different species under various conditions. As under stress conditions the miRNAs and their targets' expression changes certainly reflect plants adaptations to stresses, MIR162 $a$ and $b$ and other miRNA genes are good candidates for crops' improvement engineering experiments.

The promoter regions of both, ath-miR162a and $b$ genes, contain plenty of drought stress related response elements, as well as REs indirectly connected to salt stress via other signalling pathways. This suggests that transcriptional regulation of these genes must be already very complex. However, our previous results have shown that Arabidopsis thaliana pri-miRNA162a and pri-miRNA162b were down-regulated under drought stress, and their levels were not affected under a salinity stress (Barciszewska-Pacak et al., 2015). It suggests that transcriptional induction does not play a main role in increasing the level of the mature ath-miR162 under the stresses applied. The discrepancies between the levels of pri-miR162a, b and the mature ath-miR162 can be explained by a more efficient processing of primiRNA162a and b under drought and salinity stresses. Certainly, based on bioinformatically predicted REs in their promoter regions, this still does not exclude the transcriptional regulation of $M I R 162 a$ and $b$. The latter could be verified by determination of pri-miRNA expression and mature miRNA levels in Arabidopsis miRNA biogenesis mutants subjected to stress conditions. The pri-miRNAs' accumulation and the lowered levels of mature miRNAs could be indicative of their genes' transcriptional regulation. Furthermore, chromatin immunoprecipitation (ChIP) experiments with antibodies against RNA polymerase II could be used to show its distribution within the MIR promoter and the gene body, and to help to make conclusions about the gene transcription rate the run-on transcription experiments showing in vivo transcription rates of a gene of interest could be performed.

RNA regulatory mechanisms, including RNA synthesis, processing, transport, translation, storage, stability and degradation are emerging as key processes in modulation of stress responses (Ambrosone et al., 2012). In this context, miRNA studies in response to different stresses fit perfectly. Additionally, from the previous studies on Arabidopsis plants we concluded the pri-miRNA expression was not predictive for the level of mature miRNA (Barciszewska-Pacak et al., 2015). Thus thorough analyses of posttranscriptional miRNA gene regulation are often necessary and advisable under various conditions the plants encounter. Here, we have shown not only the existence of a complicated splicing pattern of the pri-miRNA162a under stresses applied to Arabidopsis wt plants, but also changes in the level of particular functional and non-functional splicing variants occurring under the stresses. The only functional intron retaining splicing variant appeared to be crucial for the ath-miR162a up-regulation under salinity and drought stresses, suggesting that splicing inhibition had a decisive effect on the ath-miRNA162a production under the stresses. The results proved a possible competition between splicing efficiency and miRNA production (Brown et al., 2008). So far, reports concerning the stimulatory effect of splicing on miRNAs encoded within the first exons of intron-containing miRNA genes have been published (Bielewicz et al., 2013; Schwab et al., 2013). Here, we show stimulatory effect of splicing inhibition on the intron embedded ath-miRNA162a production.
Generally, in the case of plant intronic miRNAs, the knowledge about communication between spliceosome and the plant microprocessor is limited. In 2008, the cooperation or competition between the complexes has been proposed (Brown et al., 2008). Then, for intronic miR400, a heat stress was found to activate an alternative 5' splice site (5'ss) downstream of its hairpin structure, which lowered the miR400 levels under the stress and suggested miRNA production from spliced-out introns (Yan et al., 2012). However, in the case of primiRNA162a the presence of abundant non-functional splicing variants next to the functional intron retaining variant suggests that splicing and miRNA production compete with each other.

In animals, non-canonical splicing events, alternative transcription start site, and polyadenylation site selection affect the biogenesis of miR25, miR93, miR106b (Ramalingam et al., 2014; Agranat-Tamir et al., 2014). In the case of Arabidopsis MIRs: $162 a$ and $b$, numerous polyadenylation site selections were found. This may indicate that polyadenylation, next to the splicing process, is another important level of regulation in the plant miRNA biogenesis. In the case of MIR162a, only the distal polyA isoforms are functional and were more abundant under drought but not salinity stress, when compared to the non-functional proximal polyA transcripts. Altogether, our results show that the level of ath-miR162 is regulated by posttranscriptional events like splicing and alternative polyA site selection. These results are important because of the miR162 function: miR162 controls the level of the DCL1 mRNA that is crucial for the production of almost all miRNAs in plants.

\section{Conflict of interest statement}

The authors declare no conflict of interests.

\section{Acknowledgements}

This work was supported by the NSC projects: UMO2012/04/M/NZ2/00127 and 2015/16/T/NZ1/00022, and the KNOW RNA Research Centre in Poznan, 01/ KNOW2/2014.

\section{REFERENCES}

Agranat-Tamir L, Shomron N, Sperling J, Sperling R (2014) Interplay between pre-mRNA splicing and microRNA biogenesis within the supraspliceosome. Nucleic Acids Res 42: 4640-4651. doi: 10.1093/ nar/gkt1413.

Ambrosone A, Costa A, Leone A, Grillo S (2012) Beyond transcription: RNA-binding proteins as emerging regulators of plant response to environmental constraints. Plant Sci 182: 12-18. doi: 10.1016/j.plantsci.2011.02.004.

Barciszewska-Pacak M, Milanowska K, Knop K, Bielewicz D, Nuc P, Plewka P, Pacak AM, Vazquez F, Karlowski W, Jarmolowski A, Szweykowska-Kulinska Z (2015) Arabidopsis microRNA expression regulation in a wide range of abiotic stress responses. Front Plant Sci 6: 410. doi: 10.3389/fpls.2015.00410.

Bartel DP (2004) MicroRNAs: genomics, biogenesis, mechanism, and function. Cell 116: 281-297.

Beauclair L, Yu A, Bouché N (2010) MicroRNA-directed cleavage and translational repression of the copper chaperone for superoxide dismutase mRNA in Arabidopsis. Plant J 62: 454-462. doi: 10.1111/j.1365-313X.2010.04162.x.

Bielewicz D, Dolata J, Zielezinski A, Alaba S, Szarzynska B, Szczesniak MW, Jarmolowski A, Szweykowska-Kulinska Z, Karlowski WM (2012) mirEX: a platform for comparative exploration of plant pri-miRNA expression data. Nucleic Acids Res 40: 191-197. doi: 10.1093/nar/gkr878.

Bielewicz D, Kalak M, Kalyna M, Windels D, Barta A, Vazquez F, Szweykowska-Kulinska Z, Jarmolowski A (2013) Introns of plant pri-miRNAs enhance miRNA biogenesis. EMBO Rep 14: 622-628. doi: 10.1038/embor.2013.62. 
Boyes DC, Zayed AM, Ascenzi R, McCaskill AJ, Hoffman NE, Davis KR, Görlach J (2001) Growth stage-based phenotypic analysis of Arabidopsis: a model for high throughput functional genomics in plants. Plant Cell 13: 1499-1510.

Brodersen P, Sakvarelidze-Achard L, Bruun-Rasmussen M, Dunoyer P, Yamamoto YY, Sieburth L, Voinnet O (2008) Widespread translational inhibition by plant miRNAs and siRNAs. Science 320: 11851190. doi: $10.1126 /$ science. 1159151 .

Brown JWS, Marshall DF, Echeverria M (2008) Intronic noncoding RNAs and splicing. Trends Plant Sci 3: 335-342. doi: 10.1016/j. tplants.2008.04.010.

Cheng MC, Liao PM, Kuo WW, Lin TP (2013) The Arabidopsis ETHYLENE RESPONSE FACTOR1 regulates abiotic stress-responsive gene expression by binding to different cis-acting elements in response to different stress signals. Plant Physiol 162: 1566-1582. doi: 10.1104/pp.113.221911.

Ding D, Zhang L, Wang H, Liu Z, Zhang Z, Zheng Y (2009) Differential expression of miRNAs in response to salt stress in maize roots. Ann Bot 103: 29-38. doi: 10.1093/aob/mcn205.

Dong Z, Han MH, Fedoroff N (2008) The RNA-binding proteins HYL1 and SE promote accurate in vitro processing of pri-miRNA by DCL1. Proc Natl Acad Sci USA 105: 9970-9975. doi: 10.1073/ pnas.0803356105.

Gielen H, Remans T, Vangronsveld J, Cuypers A (2012) MicroRNAs in metal stress: specific roles or secondary responses? Int I Mol Sci 13: 15826-15847. doi: 10.3390/ijms131215826.

Han MH, Goud S, Song L, Fedoroff N (2004) The Arabidopsis double-stranded RNA-binding protein HYL1 plays a role in microRNA-mediated gene regulation. Proc Natl Acad Sci USA 101: 10931098.

Higo K, Ugawa Y, Iwamoto M, and Korenaga T (1999) Plant cis-acting regulatory DNA elements (PLACE) database:1999. Nucleic Acids Res 27: 297-300.

Hirsch J, Lefort V, Vankersschaver M, Boualem A, Lucas A, Thermes C, d'Aubenton-Carafa Y, Crespi M (2006) Characterization of 43 non-protein-coding mRNA genes in Arabidopsis, including the MIR162a-derived transcripts. Plant Physiol 140: 1192-1204.

Jia F, Rock CD (2013) MIR846 and MIR842 comprise a cistronic MIRNA pair that is regulated by abscisic acid by alternative splicing in roots of Arabidopsis. Plant Mol Biol 81: 447-460. http://dx.doi. org/10.1007/s11103-013-0015-6.

Jones-Rhoades MW, Bartel DP (2004) Computational identification of plant microRNAs and their targets, including a stress-induced miRNA. Mol Cell 14: 787-799.

Kruszka K, Pacak A, Swida-Barteczka A, Nuc P, Alaba S, Wroblewska Z, Karlowski W, Jarmolowski A, Szweykowska-Kulinska Z (2014) Transcriptionally and post-transcriptionally regulated microRNAs in heat stress response in barley. J Exp Bot 65: 6123-6135. doi: $10.1093 / j x b /$ eru353.

Kruszka K, Pacak A, Swida-Barteczka A, Stefaniak AK, Kaja E, Sierocka I, Karlowski W, Jarmolowski A, Szweykowska-Kulinska Z (2013) Developmentally regulated expression and complex processing of barley pri-microRNAs. BMC Genomics 14: 34. doi:10.1186/14712164-14-34.

Kurihara Y, Takashi Y, Watanabe Y (2006) The interaction between DCL1 and HYL1 is important for efficient and precise processing of pri-miRNA in plant microRNA biogenesis. RNA 12: 206-212.

Laubinger S, Sachsenberg T, Zeller G, Busch W, Lohmann JU, Ratsch $G$, Weigel D (2008) Dual roles of the nuclear cap-binding complex and SERRATE in pre-mRNA splicing and micro RNA processing in Arabidopsis thaliana. Proc Natl Acad Sci USA 105: 8795-8800. doi: $10.1073 /$ pnas.0802493105.

Liu J-X, Srivastava R, Che P, Howell SH (2007) Salt stress responses in Arabidopsis utilize a signal transduction pathway related to endoplasmic reticulum stress signalling. Plant J 51: 897-909.

Lobbes D, Rallapalli D, Schmidt DD, Martin C, Clarke J (2006) SERRATE: a new player on the plant microRNA scene. EMBO Rep 7: 1052-1058.

Lu SF, Sun YH, Shi R, Clark C, Li LG, Chiang VL (2005) Novel and mechanical stress responsive microRNAs in Populus trichocarpa that are absent from Arabidopsis. Plant Cell 17: 2186-2203.

Manavella PA, Hagmann J, Ott F, Laubinger S, Franz M, Macek B, Weigel D (2012) Fast-forward genetics identifies plant CPL phosphatases as regulators of miRNA processing factor HYL1. Cell 151: 859-870. doi: 10.1016/j.cell.2012.09.039.
Megraw M, Baev V, Rusinov V, Jensen ST, Kalantidis K, Hatzigeorgiou AG (2006) MicroRNA promoter element discovery in Arabidopsis. RNA 12: 1612-1619. doi: $10.1261 /$ ma.130506.

Park W, Li J, Song R, Messing J, Chen X (2002) CARPEL FACTORY, a Dicer homolog, and HEN1, a novel protein, act in microRNA metabolism in Arabidopsis thaliana. Curr Biol 12: 1484-1495.

Ramalingam P, Palanichamy JK, Singh A, Das P, Bhagat M, Kassab MA, Sinha S, Chattopadhyay P (2014) Biogenesis of intronic miRNAs located in clusters by independent transcription and alternative splicing. RNA 20: 76-87. doi: 10.1261/rna.041814.113.

Reinhart BJ, Weinstein EG, Rhoades MW, Bartel B, Bartel DP (2002) MicroRNAs in plants. Gene Dev 16: 1616-1626.

Ren G, Xie M, Dou Y, Zhang C, Yu B (2012) Regulation of miRNA abundance by RNA binding protein TOUGH in Arabidopsis. Proc Natl Acad Sci USA 109: 12817-2821. doi: 10.1073/pnas.1204915109.

Rogers K, Chen X (2013) Biogenesis, turnover, and mode of action of plant microRNAs. Plant Cell 25: 2383-2399. doi: http://dx.doi. org/10.1105/tpc.113.113159.

Schwab R, Speth C, Laubinger S, Voinnet O (2013) Enhanced microRNA accumulation through stemloop-adjacent introns. EMBO Rep 14: 615-621. doi: 10.1038/embor.2013.58.

Sun G, Stewart CN Jr, Xiao P, Zhang B (2012) MicroRNA expression analysis in the cellulosic biofuel crop switchgrass (Panicum virgatum) under abiotic stress. PLOS ONE 7: e32017. doi: 10.1371/journal. pone.0032017.

Szarzynska B, Sobkowiak L, Pant BD, Balazadeh S, Scheible WR, Mueller-Roeber B, Jarmolowski A, Szweykowska-Kulinska Z (2009) Gene structures and processing of Arabidopsis thaliana HYL1-dependent pri-miRNAs. Nucleic Acids Res 37: 3083-3093. doi: 10.1093/ nar/gkp189.

Szweykowska-Kulinska Z, Jarmolowski A, Vazquez F (2013) The crosstalk between plant microRNA biogenesis factors and the spliceosome. Plant Signal Behav 8: e26955. http://dx.doi.org/10.4161/ psb. 26955.

Vazquez F, Gasciolli V, Crete, P, and Vaucheret H (2004) The nuclear dsRNA binding protein HYL1 is required for microRNA accumulation and plant development, but not posttranscriptional transgene silencing. Curr Biol 14: 346-351.

Wang L, Song X, Gu L, Li X, Cao S, Chu C, Cui X, Chen X, Cao X (2013) NOT2 Proteins Promote Polymerase II-Dependent Transcription and Interact with Multiple MicroRNA Biogenesis Factors in Arabidopsis. Plant Cell 25: 715-727. doi:10.1105/tpc.112.105882.

Xie Z, Kasschau KD, Carrington JC (2003) Negative feedback regulation of Dicer-Like1 in Arabidopsis by microRNA-guided mRNA degradation. Curr Biol 13: 784-789.

Xie Z, Allen E, Fahlgren N, Calamar A, Givan SA, Carrington JC (2005) Expression of Arabidopsis miRNA genes. Plant Pbysiol 138: 2145-2154.

Yan K, Liu P, Wu CA, Yang GD, Xu R, Guo QH, Huang JG, Zheng CC (2012) Stress-induced alternative splicing provides a mechanism for the regulation of microRNA processing in Arabidopsis thaliana. Mol Cell 48: 521-531. http://dx.doi.org/10.1016/j.molcel.2012.08.032.

Zhan X, Wang B, Li H, Liu R, Kalia RK, Zhu JK (2012) Arabidopsis proline-rich protein important for development and abiotic stress tolerance is involved in microRNA biogenesis. Proc Natl Acad Sci USA 109: 18198-18203. doi:10.1073/pnas.1216199109.

Zhang Z, Wei L, Zou X, Tao Y, Liu Z, Zheng Y (2008) Submergence-responsive microRNAs are potentially involved in the regulation of morphological and metabolic adaptations in maize root cells. Ann Bot 102: 509-519. doi: 10.1093/aob/mcn129.

Zhao X, Zhang H, Li L (2013) Identification and analysis of the proximal promoters of microRNA genes in Arabidopsis. Genomics 101: 187-194. http://dx.doi.org/10.1016/j.ygeno.2012.12.004.

Zhou L, Liu Y, Liu Z, Kong D, Duan M, Luo L (2010) Genome-wide identification and analysis of drought-responsive microRNAs in Oryza sativa. J Exp Bot 61: 4157-4168. doi: 10.1093/jxb/erq237.

Zielezinski A, Dolata J, Alaba S, Kruszka K, Pacak A, Swida-Barteczka A, Knop K, Stepien A, Bielewicz D, Pietrykowska H, Sierocka I, Sobkowiak L, Lakomiak A, Jarmolowski A, Szweykowska-Kulinska Z, Karlowski WM (2015) mirEX 2.0 - an integrated environment for expression profiling of plant microRNAs. BMC Plant Biol 15: 144. doi: 10.1186/s12870-015-0533-2. 